\title{
An automated solution for fixtureless sheet metal forming
}

\author{
Balaji Ilangovan $^{1,2} \cdot$ Radmehr P. Monfared ${ }^{1,2}$. \\ Michael Jackson $^{1,2}$
}

Received: 15 December 2014 / Accepted: 21 May 2015 / Published online: 11 June 2015

(C) The Author(s) 2015. This article is published with open access at Springerlink.com

\begin{abstract}
Manual forming of sheet metal parts through traditional panel beating is a highly skilled profession used in many industries, particularly for sample manufacturing or repair and maintenance. However, this skill is becoming gradually isolated mainly due to the high cost and lack of expertise. Nonetheless, a cost-effective and flexible approach to forming sheet metal parts could significantly assist various industries by providing a method for fast prototyping sheet metal parts. The development of a new fixtureless sheet metal forming approach is discussed in this article. The proposed approach, named Mechatroforming ${ }^{\circledR}$, consists of integrated mechanisms to manipulate sheet metal parts by a robotic arm under a controlled hammering tool. The method includes mechatronicsbased monitoring and control systems for (near) real-time prediction and control of incremental deformations of parts. This article includes description of the proposed approach, the theoretical and modelling backgrounds used to predict the forming, skills learned from manual operations, and proposed automation system being built.
\end{abstract}

Keywords Automation $\cdot$ Flexible manufacturing $\cdot$ Rapid prototyping $\cdot$ Incremental forming

Balaji Ilangovan

B.Ilangovan@lboro.ac.uk

1 EPSRC Centre for Innovative Manufacturing in Intelligent Automation, Loughborough University, Loughborough, UK

2 Wolfson School of Mechanical and Manufacturing Engineering, Loughborough University, Loughborough, UK LE11 3TU

\section{Introduction}

In many industries, dies are used for forming sheet metals but they typically lack flexibility and cost-effectiveness when low-volume production or prototyping are considered. For these cases, the traditional manual panel beating is still used. However, manual panel beating is a highly skilled process and unfortunately, due to the lack of interest (by new trainees), high cost, and sporadic industrial applications, is becoming isolated and gradually being lost.

There is not enough research carried out to fully understand and capture the skills of experienced panel beaters and its links with the formal sheet metal forming theories.

Analysing the conventional manual practises has led to an ongoing research work in robotic sheet metal forming which has been discussed in this article. The proposed research methodology could potentially be used by many industries, especially for maintenance and rapid prototyping of sheet metal parts.

There has been some research in die-less incremental forming of sheet metals [1]. Most proposed methods use either stretching or drawing techniques with or without an anvil support. The sheet metal is formed by using a round headed tool which moves down vertically (i.e. in $Z$ axis). The sheet metal is typically held by fixtures along the edges to avoid movement caused by tool. Either the sheet metal or the tool is moved along $X Y$ axes (i.e. the horizontal plane) to achieve the required contour. The forming is often computer controlled. Employing this technique achieves a smooth finish but may result in fractures due to excessive stretching and often fails while forming complex contours (e.g. having more than $55^{\circ}$ wall angle [2]). These methods use fixtures to hold the sheet and typically result in non-uniform thickness of the formed sheet metal, hence, affecting the quality. 
The use of fixtures limits the part complexity and size dramatically. They also apply additional forces to the forming process that result in reducing the thickness (via nonuniform stretching) and changes the physical properties and dimensions in an almost unpredictable manner (e.g. the spring back action). The manual forming process through the traditional panel beating can increase the uniformity in thickness of the material and through incremental forming and inspection processes minimises unplanned deformation of parts due to residual stresses or forming errors.

An ongoing research in Loughborough University on developing a new automated incremental sheet metal forming is aimed at understanding, capturing and automating manual fixtureless sheet metal forming process. The proposed approach focusses on (a) eliminating the need for fixtures and (b) automating based on replicating manual forming processes used by skilled operators.

By closely observing and analysing the human skills of experienced panel beaters, it is perceived that consecutive shrinking and stretching (through hammering) of sheet metal will allow the workpiece to have uniform thickness while being formed. Imposing repetitive kinetic energy through hammering allows better control over producing shapes incrementally by consecutive stretching and shrinking without fixtures. This method is also believed to improve release of residual stresses during the forming process.

It was observed in a manual process that to achieve the necessary forming, an impact force or kinetic energy will have to be imposed on the point of forming at certain angle. The shape and the angle of this reflected force will have significant influence on the material while forming. However, angular impact will introduce further complexity in an automated forming mechanism.

The proposed method discussed in this article includes a mechanised hammer used to form 3D freeform geometry of sheet metal parts incrementally while the sheet metal is held by a robot in a fixtureless environment. The incremental forming is controlled and monitored by understanding and using human factors from manual operations and finite element analysis (FEA modelling) for pre-processing analysis and by implementing 3D shape measurement technology and force sensors for real-time and post-processing analysis.

\section{Existing approaches and factors involved in incremental forming}

Sheet metal forming involves several design and operational factors before, during and after the formation of parts. These include both product and process design factors. From uncoiling of the sheet metal to monitoring the forming, each step is significant to achieve the required forming.
A number of commercial technologies and research methodologies for incrementally forming sheet metals have been analysed. Traditional sheet metal forming by panel beaters has also been perceived and carefully analysed. The existing approaches in incremental forming are divided into the following:

- The product and process design factors affecting the forming of material

- Traditional manual forming methods

- Automated dieless sheet metal forming processes

- Current state of the art in research (impact forming)

\subsection{Product and process design factors}

There are certain product and process design factors which affects the sheet metal forming. For instance, material properties of the sheet metals such as its crystallographic structure and thickness influence its forming [3].

Conforming to the material aspects, the sheet metal is typically cut along the edges to produce the initial 2D contour to start the forming process, but this may lead to fracture. Hence, the initial 2D contour is laser cut after generating the initial geometry through reverse engineering either by stereo lithography (STL) geometrical modelling [4] or designed using an expert computer-aided process planning (CAPP) system [5]. Beginning with the forming process, it is suggested the forming should adhere to limitation of stress/strain index and a material's forming limit might be based on forming limit curve (FLC) for maximum limits or forming fracture limit (FFL) for fracture limits [6].

While forming the material incrementally, it is essential to account for the development of residual stress [7] and spring back behaviour influenced by plastic modulus of stress/strain response as they may cause deviations in the forming process. To realise the relation between impact force and deformation, it is vital to understand the kinetic energy involved in deforming the material and dissipation of maximum kinetic energy onto workpiece [8].

Once these factors have been considered, the final steps in incremental forming are planning the path of forming and monitoring using feedback systems, typically done by 3D shape measurement and comparing the formation with $\mathrm{CAD}$ model of the final contour [9].

\subsection{Traditional manual forming}

The manual forming as a conventional method of sheet metal forming was analysed by observing experienced panel beaters with the aim of capturing the skills and applying to the automated approach. In manual forming, the parts are formed 
using hammer and dollies. The sheet metal is held between the hammer and dolly. The dolly is typically held stationary, and hammer is used to apply impact repetitively at the same point with gradual movement of the part. The part is moved gradually and skewed over the dolly to produce the required contour. The methodology and equipment for forming are almost common for any contour required. Initially tucks (larger impact force resulting in larger deformation) were formed to introduce the basic shape required. The tucks were formed with reasonable gaps to avoid affecting the earlier formed tucks. Constructing on the formed tucks, the sheet metal is shrunk and stretched consecutively to produce the required contour. The sheet metal is held in hand while forming, which also acts as a damper bearing the vibration produced on the work piece. The damping action enhances the dissipation of kinetic energy on the sheet metal and allows better prediction of deformation based on the impact force applied. Figure 1 illustrates the double curvature forming technique.

\subsection{Automated dieless sheet metal forming}

Incremental sheet metal forming is practiced by many industries in commercial sector $[10,11]$, and therefore, there has been an extensive research in this field.

There has been a significant research done and being carried out in stretching methods including single-point incremental forming (SPIF) [12] and double-point incremental forming (DPIF) [13]. Though they were studied, they are beyond the scope of this research. Researches related to impact forming have been discussed in detail in Sect. 2.3.1.

\subsubsection{Research—impact forming}

As sheet metals are produced traditionally using manual hammering, there have been very few researches aiming at forming the sheet metal using hammering (impacting).

Tanaka [14] proposed a linear servo hammering unit using linear servo motor. It was suggested by this research that most of the kinetic energy was used for deforming the material rather shaping the material to required contour. It was found that successive impacts were partially sensitive to the region of earlier impacts and can influence the deformation achieved. Schafer [15] introduced eccentric cam to the hammering method with two counter balancing masses to balance the masses. The sheet metal was formed by means of punches produced by the hammering unit reciprocating due to the eccentricity of the cam.

Both the above discussed techniques used motion of tool to achieve the required contour ( $2 \mathrm{D}$ motions by a robot in the servo mechanism and six axis robot in the eccentric cam mechanism) while the sheet metal was held in a fixture. Forming the sheet metal by motion of tool has got a significant effect in forming since the vibration caused may affect the hammering unit and may cause inaccuracy in forming. The sensitivity of forming during the successive impacts discussed by Tanaka is actually due to the residual stresses formed after the earlier impact. The effects of these residual stresses have been discussed in detail in Sect. 4 of this article.

Replacing the motion of tool, Opritescu [16] introduced 'kraftformer' for shrinking the edges of the sheet metal using a shrinking machine and a six axis robot which held the sheet metal. The sheet metal was driven by the robot in the path that were tracked and imparted from human motions of shrinking the sheet metal. In this method, tests were made only to shrink the sheet metal on the edges by kraftformer which measured $6 \%$ deviation to that shrunk by human.

Furthermore, CoTesys [17] in Germany have been researching on capturing the cognitive knowledge to form the sheet metal using driving methodology [18]. The research named 'CoDrive' has provided a significant contribution towards incremental sheet metal forming using the kraftformer. Incremental impact stretch forming was implemented using the kraftformer to form the sheet metal which was held by the robot. The research was aimed at understanding on how the robot motion alters itself to the geometrical changes (especially the bending radii) in the sheet metal while stretching. Though a significant contribution was made, the research was confined to producing a $2 \mathrm{D}$ contour using stretching and did not involve forming 3D freeform geometry using simple impact, which is discussed in this article.
Fig. 1 Traditional manual forming
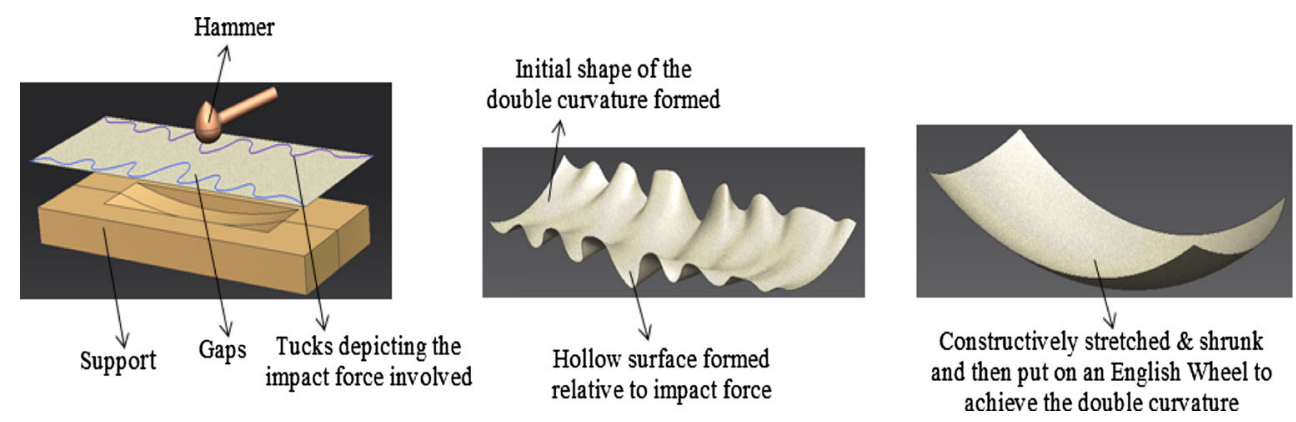


\subsection{Comparison between stretching and automated hammering}

Comparing the incremental stretching and hammering techniques, a study carried out by researchers in Cambridge University has found that hammering process had a steady impulse response in comparison to incremental stretch forming [19]. It was also found that hammering is better for globalised forming rather using incremental stretching as it tends to be more localised. Higher wall angles and deeper curvature could not be produced using stretching process as it often leads to fracture of the material. Nonetheless, hammering creates a noisy environment and for equally smoother surface further process may be required (e.g. planishing).

\section{Mechatroforming}

Based on the observation of manual panel beating by skilled labours and analysing the different factors affecting the sheet metal forming, the Mechatroforming method was developed and tested for fixtureless incremental forming as part of the research reported in this article. As shown in Fig. 2, a robot holds the sheet metal similar to manual operator and the hammering unit forms the sheet by impacting the sheet which is held upon a support. The support will be universal to provide flexibility in forming different contours. The robot provides the motion and angle for incrementally forming the sheet metal under the hammer.

\subsection{Proposed methodology}

Based on the requirements for predicting the forming of sheet metal, a mechatronics based control system was developed as shown in Fig. 3. This system enables a (near) real time monitoring of forming following each impact to configure the impacting pattern accordingly to achieve the required shape of the sheet metal.

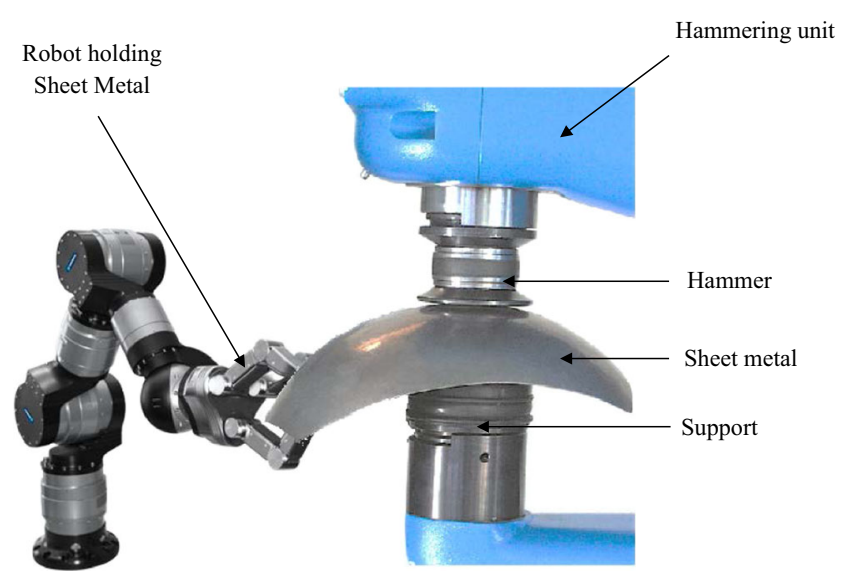

Fig. 2 Mechatroforming approach
The robot and the hammering unit are integrated using the following components:

- Database - consisting of human skills captured and preprocessing FEA analysis

- Processor - for processing the data being monitored and reconfiguring impact pattern relative to the current state of the system

- Robot controller - to control the motion of sheet metal to achieve the shape required

- Integrated force controller - to control the motion of hammer to achieve the deformation required

- 3D shape measurement - to monitor the forming of desired shape and deformation

- Force sensor - to measure and predict the deformation achieved after each impact

The skills observed from manual operator are interpreted as system parameters and fed into the database. These system parameters include (a) clearance between the surface of forming and surface where the sheet metal is held, (b) grain direction in which the forming is started, (c) pattern in which the required shape could be easily achieved, (d) space in between the initial impacts to achieve the primary shape, and (e) co-ordinate points according to which the sheet will have to be moved and skewed. The data is fed based on the initial few impacts required to achieve the primary shape and the consecutive shrinking and stretching needed to achieve the final desired shape. The process mentioned above is simulated using FEA and used for preprocessing analysis. Significant steps in achieving the required contour will be predicted based on the FEA modelling and manual process pattern. Constructing on these steps, hammering path will be planned to form the sheet metal to the desired contour. This path is then fed as input to the system to initiate the iterations in forming the sheet metal. The path information fed as input will be based on the depth of forming at each impact point.

Based on the iterations that are continuously monitored and corrected, the robot controller manipulates the robot to locate the sheet metal to the required position and orientation between hammer and the support. The FEA model specifies the magnitude of force for each impact. Accordingly, the integrated force controller specifies the force of the hammer required to achieve the necessary deformation. This results in hammer impacting the sheet metal to produce a deformation.

Once each impact is made, the force sensor provides the real-time measure of the impact force. The deformation achieved is calculated after each impact is found from the measured impact force. This information is then fed back into the control system based on which the processor optimises the next impact. 


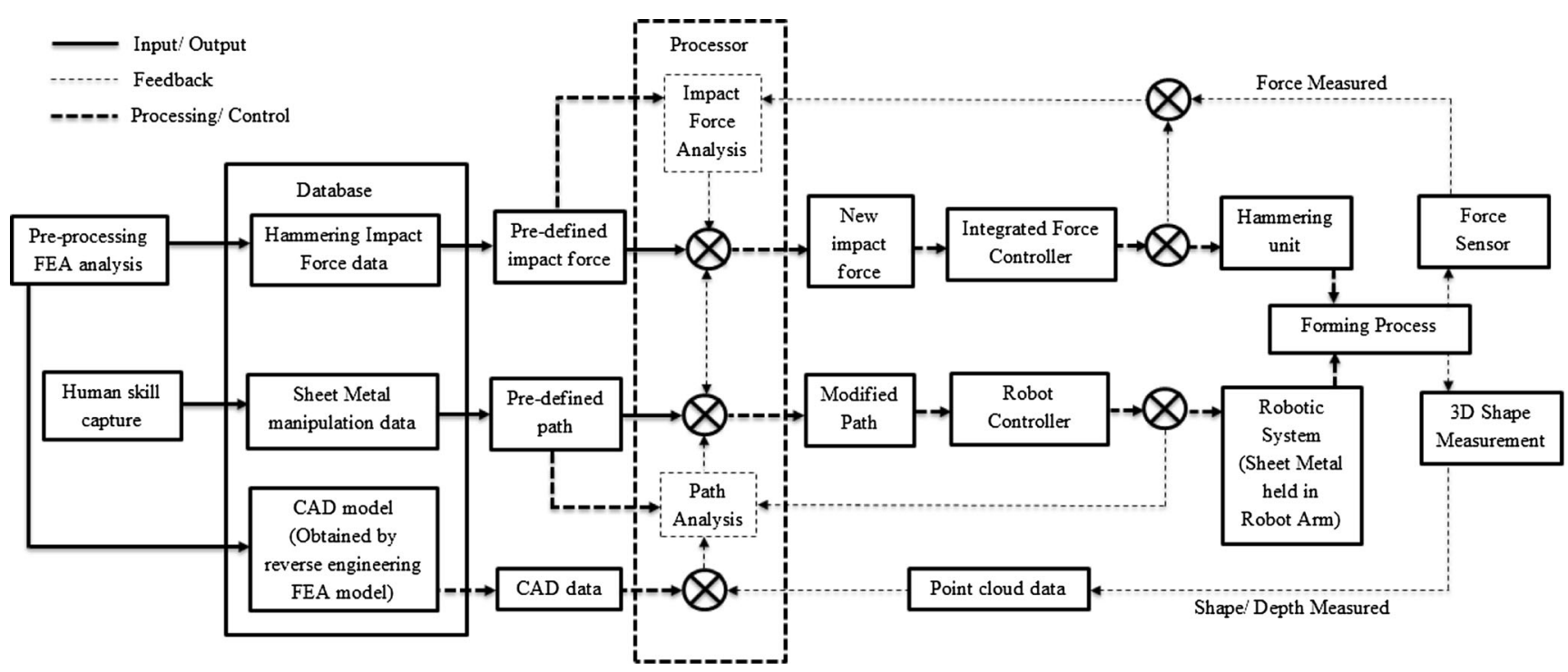

Fig. 3 Control system for Mechatroforming

After each sets of impact, 3D shape measurement system scans the sheet metal to create a point cloud data with depth and shape information about the formed contours. The point cloud data is then fed back into the control system to be compared with the desired contour which is usually a CAD model of the final shape required.

With the existing arrangement of the prototype system, it is rather a time-consuming process to scan the part after moving it away from the hammering unit. However, it is anticipated that the process is optimised to be faster by refining the impact steps based on the measured impact force and the calculated displacement after each impact.

The iterations continue until the desired shape of the sheet metal is achieved as compared to CAD model of the same.

\subsection{The process of Mechatroforming}

Primarily, the initial contour to start the forming is designed by reverse engineering the CAD model of final shape to be achieved.

The initial process has tucks being formed to produce the basic formability shape of the required contour. The location and force of the first impact will be based on the combined database of FE analysis and system parameters observed from human skills (referred as system parameters here). Once the first impact has been made, impact force sensor will feedback the impact force to predict the deformation achieved. The preprocessing FE analysis will provide the region sensitive to residual stresses because of that impact; hence, the next impact could be made away from the sensitive region based on the system parameters.

After the initial formable shape is achieved, the consecutive shrinking and stretching will lead to the contour required. The pre-processing FE analysis and the system parameters combined will generate contour slices based on which the sheet metal will have to be formed. Each contour slice will follow a perceived path and will need to achieve the required shape and deformation.

The 3D shape measurement scans the sheet metal and produces the depth and the shape information that is fed back into the control system. The achieved contour will be compared to the pre-defined path. Any error in the path will be rectified by the feature detection algorithm developed in the control system. The algorithm will make the decision considering the initial pre-defined contour path, current contour of the sheet metal and the final contour to be achieved. Once the error has been rectified, the usual process is continued until the final shape has been achieved.

\section{Control characteristics of Mechatroforming}

Mechatroforming requires control based on its characteristics as illustrated in Fig. 4.

The primary control characteristic of Mechatroforming is to grip the sheet metal by means of a robotic gripper. Adaptability of the gripper is essential to adjust relative to the continuously changing contour. The gripper and robot will have to bear the oscillation or vibration caused due to a dynamic impact. Failing to damp will affect the gripper as well as dissipation of kinetic energy in the sheet metal. Therefore, a damper is provided at the intersection of gripper and the sheet metal. Damping is applied by having a flexible gripper and softer gripping material. The hammer material is also chosen appropriately such that it complements the damping action and also imparts the force required.

The robot manipulates the sheet metal to locate it in the required position and orientation between the hammer and the 
Fig. 4 Control characteristics of Mechatroforming

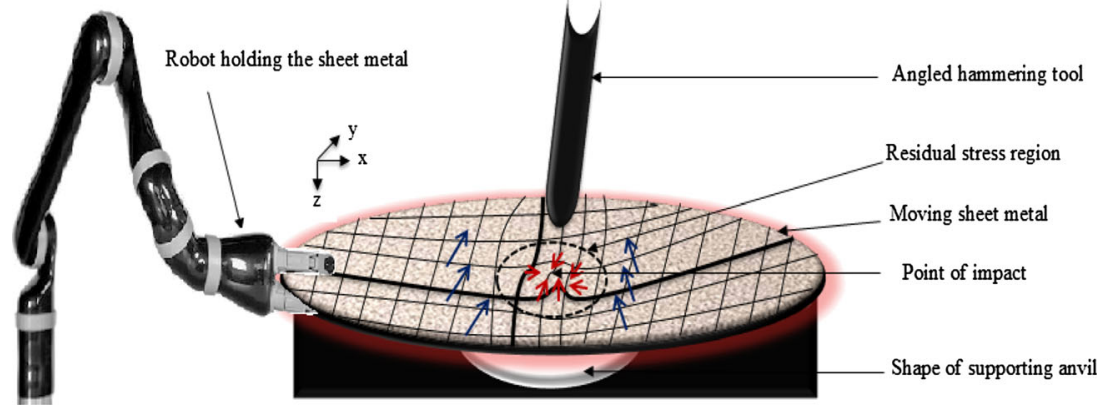

support. The sheet metal will have to be moved along the $X Y$ plane and skewed about the $X$ or $Z$ axis (refer to Fig. 4) according to the necessity. This technique is inferred from the manual panel beating method and provides higher formability.

Though skewing the sheet provides a better control, sometimes angled tooling will be required to achieve the required shape. As the angled tooling introduces computational complexity, this is also provided by orientation of parts. If the sheet is not skewed to an appropriate angle, all the energy dissipated might go into deforming the sheet metal rather shaping it to required contour.

Designing the size and shape of the support is important to determine the deformation achieved as it has direct influence to the impact force applied on the sheet metal. Through experiments, it is anticipated that reducing the size of support, the deformation achieved will be more predictable and the shape of deformation can be well defined as the region of reaction force will be reduced.

Soon after an impact, residual stresses get developed around the point of impact. These residual stresses cause changes to the material properties. The sheet metal is analysed both locally and globally before making the next impact as it is sensitive to the residual stress region and will have consecutive effect in forming the sheet metal. It is difficult to feedback the material properties of the sheet metal after each impact in real time. This requires high computations and longer time duration which is commercially not viable. Therefore, the monitoring process should be carried out frequently in accordance with the system's computational processing power. This was also experienced through the manual panel beating and applied using the system parameters.

\section{Experimental research}

It was understood that incremental deformation achieved on the sheet metal relative to the impact force will have to be determined in terms of kinetic energy applied within a short period of time.
Therefore, it was necessary to perform a time based analysis to find the kinetic energy required for deforming the sheet metal during each impact. This analysis was also necessary to develop a system to control the impact forces based on time scales.

It was also necessary to validate the theoretical findings as the impact occurs for a very short duration of about $0.2 \mathrm{~s}$. Therefore, an instrumented hammer with an embedded force transducer was used to measure the impact force in this test.

Both these experiments were concurrently performed. As the deformation achieved would be same, the impact force determined in each case could be compared for validation.

The FEA model was developed to provide preprocessing analysis to determine the impact force based on the deformation that should be achieved similar to the previous experimental method. The objective was to ensure that FEA results align with the results achieved using time-based analysis and instrumented hammer. In which case, FEA could be used to simulate the process and predict the forming at each contour monitoring stage combined with the analysis obtained from 3D shape measurement system.

\subsection{Experimental set-up}

All the experiments were performed on aluminium of 1-mm thickness and 275-mm diameter. The sheet was held in circular wooden block, hollow at its centre to about 135-mm diameter. In the case of FEA, a similar set-up was made virtually.

In the first experiments, the instrumented hammer was used to calculate the impact force as shown in Fig. 5. The crosssectional view of the support used is illustrated in Fig. 6. The hammer has a force transducer at its tip which has a sensitivity of $0.499 \mathrm{mV} \mathrm{N}^{-1}$. The impact force was calculated by amplifying the signal using the charge amplifier and reading the output voltage using an oscilloscope.

While impacting the sheet metal with impact hammer, time-based analysis was also carried out by using a high-speed camera. With $5 \times 5$-cm black and white grid lines $800 \mathrm{~mm}$ high at the backdrop, the hammering action was captured at 1000 frames per second. Total of 
Fig. 5 Experimental set-up

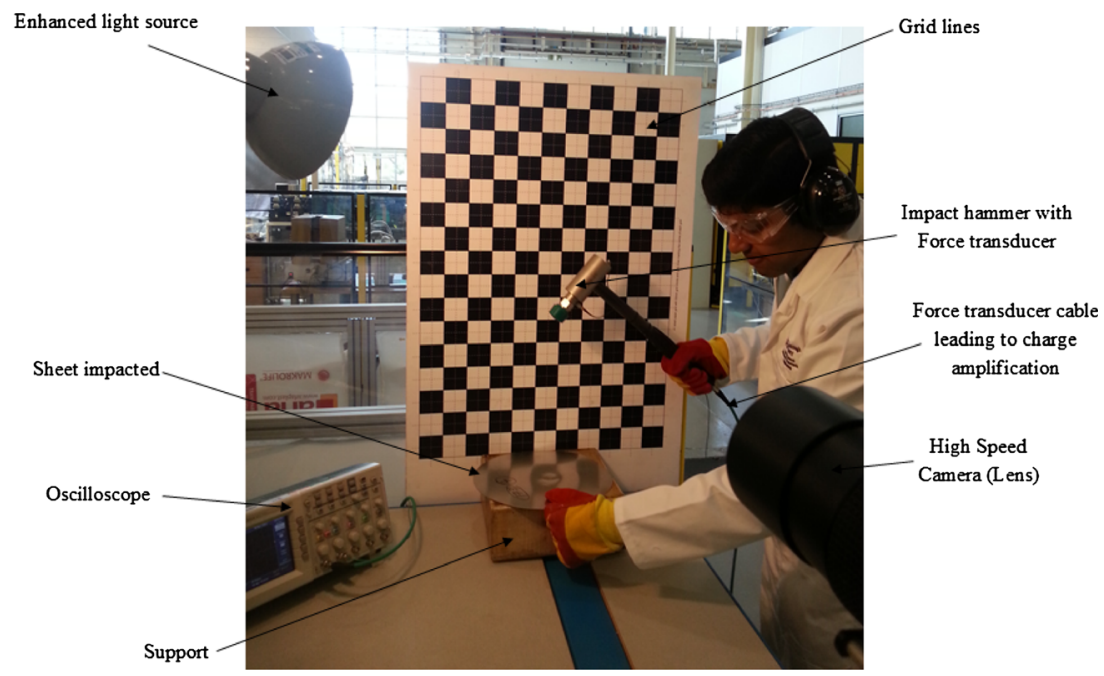

33 data points were collected while the hammer moved from $800-\mathrm{mm}$ distance to 0.00 datum plane where it impacted the sheet. These time and distance data were interpreted in Matlab software to find the fourth-order polynomials. The formula used is given as follows [20]:

$P(x)=P_{1} x^{n}+P_{2} x^{n-1}+\ldots+P_{n} x+P_{n+1}$

Finds the coefficient of polynomial $P(x)$ of degree $n$.

Since the motion of the hammer is accelerated, the velocity and acceleration of the hammer are variable with time. Hence, calculations were made based on time-dependent acceleration. The equations for calculations were based on the following [20]:

Position, $x(t)=x_{0}+v_{0} t+b \frac{t^{2}}{2}+c \frac{t^{3}}{6}+d \frac{t^{4}}{12}=x_{0}+\int_{0}^{t} v \partial t(2)$

Velocity, $\frac{\partial x}{\partial t}=v(t)=v_{0}+b t+c \frac{t^{2}}{2}+d \frac{t^{3}}{3}=v_{0}+\int_{0}^{t} a \partial t(3)$

Acceleration, $\frac{\partial x^{2}}{\partial t^{2}}=a(t)=b+c t+d t^{2}$

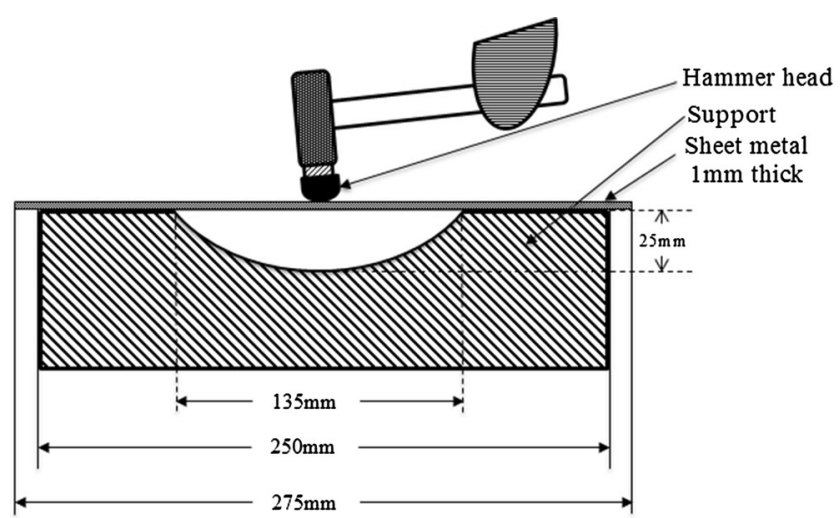

Fig. 6 Cross-sectional view
Using Eq. 3, the impact velocity of the hammer at the point of impact could be found. The impact kinetic energy was found using the following equation:

$K \cdot E=\frac{1}{2} m V^{2}$

where $m$ is the mass of the hammer used and $V$ is the impact velocity of the hammer.

From the above illustration, it could be noted that kinetic energy K.E delivered to the sheet metal is directly proportional to the distance travelled by the hammer after impact, $d$. Energy lost by friction due to air and impact are negligible as they have very minor error factor compared to the larger impact force. The hammer's deceleration effect and material displacement effect have been neglected as energy delivery is not the prime scope of this research. The consistency of manual hammering was calibrated in advance by practicing a uniform hammering on different work pieces.

Equating kinetic energy is equivalent to work done:

$K . E=F \times d$

The force, $F$, calculated will be the impact force.

As the above discussed theory is applied in static loading, theory behind the dynamic impacts was also studied. Based on the hertz theory of contact [21], there is some elastic energy transferred as vibration in the sheet metal during dynamic impact, it is necessary to find this vibrational energy to determine the actual kinetic energy spent in deforming the sheet metal. Calculation of vibrational energy is by means of pressure pulse, and it depends on the Poisson's ratio and mass density of both the hammer and the sheet metal. Then, the equation could be related as,

$K . E$-vibrational energy $=F \times d$ 
During experiments, the energy calculated in comparison to the deformation achieved suggested that vibrational energy has no significant influence on the energy delivered if the sheet was held securely. Hence, the vibrational energy was considered to be negligible considering the scope of the experiments.

In addition, some of the data regarding the material mechanics generated by the FEA model was also considered to be beyond the scope of this experiment and therefore was disregarded. It was envisaged that such assumption will have negligible impacts on the results of the experiments. The prime purpose was to develop a comparative time based analysis, and it was proved that the Eq. 6 was adequate since the impact force was also measured in real time using an instrumented hammer.

\subsection{Finite element analysis}

The FEA modelling was used to predict the incremental deformation and determine the next set of forming. Hence, the entire experiment was simulated using the FEA and the results were fed as a pre-processing input to the system in determining the forming pattern.

The FEA modelling was performed using MSC MARC software as it was understood to be the efficient software in determining time-based dynamic impacts. In addition, it was the software application available to this research. The FE analysis was carried out for the same set of data as used in the previous manual experiments. All the material properties were specified more realistically in the simulation model using the pre-defined parameters defined by the software. The dimensions of the sheet and the hammer were given relatively as FEA is a dimensionless system.

To develop the model, the geometrical properties, meshing and material properties were mentioned for the aluminium sheet used for the experiments. The hammer was defined as a rigid body (considering negligible deformation to its body). The contact conditions between the hammer and the sheet were timed similarly to the timing results obtained from the high-speed camera test. The material was constrained similar to the practical set up in which a circular hollow support is used. The aluminium's plasticity was determined by performing dog bone tests on the material used for previous experimental purposes. This was later fed as stress/strain curve into the FEA model as it would give more realistic material inputs to perform the analysis.

A deformation-based analysis was carried out using the FEA model. The hammer was held at a height of $D$ and was indicated to travel a distance of $(D+d)$ deforming the object to distance, $d$, relatively similar to the one produced in practical experiments (refer to Fig. 7). The time hammer took to deform the material was specified based on the time hammer travelled to the distance $(D+d)$ in practical experiments (refer to Table 1). By running the model, the impact force required to achieve the expected deformation was determined. Results of the above experiments have been discussed below.

\subsection{Experiments}

On performing the experiments by using the set-up discussed in Sects. 5.1 and 5.2, the following results were achieved.

\subsection{Experiment 1}

The set-up was similar to the one discussed in Sect. 5.1, and the impact hammer of mass $1 \mathrm{~kg}$ was used to impact the sheet metal. The hammer travelled $0.212 \mathrm{~s}(t)$ for a distance of $0.8065 \mathrm{~m}(D+d)$ producing a deformation with depth $(d)$ of $0.0065 \mathrm{~m}$. The impact velocity of the hammer was determined by the high-speed camera and was calculated using Eq. 3 as $7.17 \mathrm{~ms}^{-1}$. The impact kinetic energy was calculated using Eq. (5) as $25.70 \mathrm{~J}$. The impact force was calculated using Eq. 6 as $3954.5 \mathrm{~N}$.

Similarly, using the impact hammer, a voltage of $2.08 \mathrm{~V}$ (refer Fig. 8-Exp1) was measured upon impact. Calculating the impact force based on the sensitivity of $0.499 \mathrm{mV} \mathrm{N}^{-1}$, the impact force was calculated as $4168 \mathrm{~N}$.
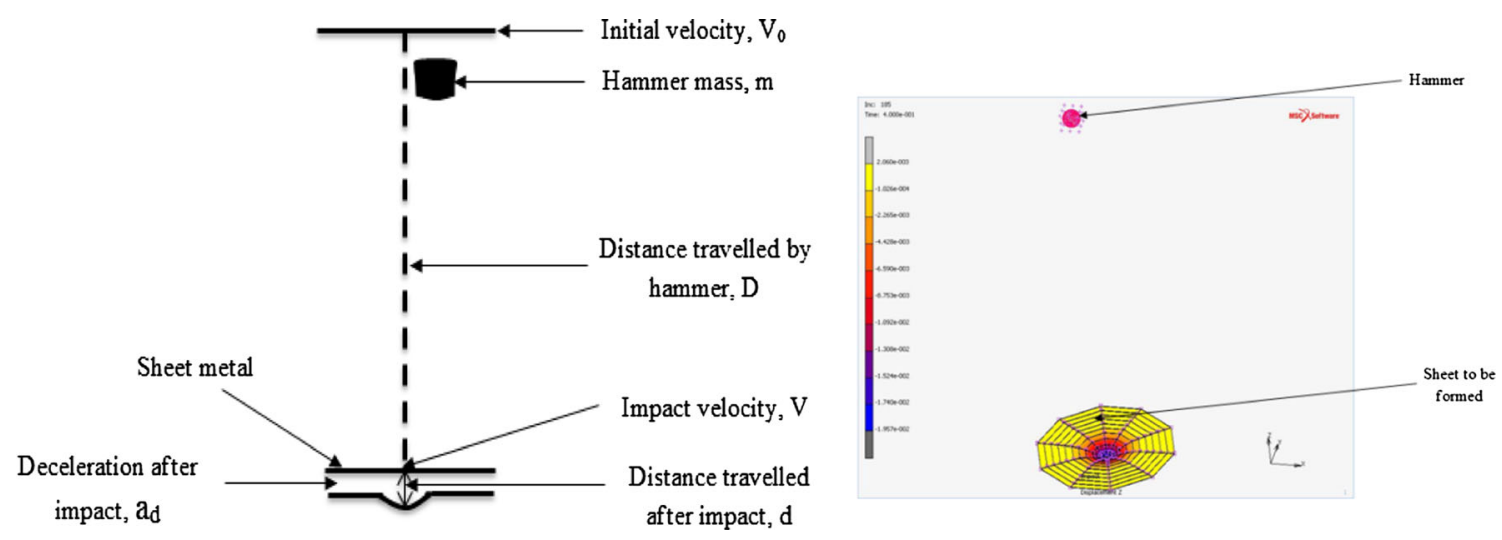

Fig. 7 Theoretical calculation of impact force and FEA simulation 
Table 1 Results achieved

\begin{tabular}{|c|c|c|c|c|c|c|c|c|c|c|}
\hline \multirow[t]{2}{*}{$\begin{array}{l}\text { Experiment } \\
\text { no. }\end{array}$} & \multirow{2}{*}{$\begin{array}{l}\text { Time travelled } \\
\text { by hammer } \\
\text { (s) }\end{array}$} & \multirow{2}{*}{$\begin{array}{l}\text { Distance travelled } \\
\text { by hammer }(D+d) \\
\text { (m) }\end{array}$} & \multirow{2}{*}{$\begin{array}{l}\text { Deformation } \\
\text { achieved }(d) \\
(\mathrm{m})\end{array}$} & \multicolumn{2}{|c|}{$\begin{array}{l}\text { Instrumented impact } \\
\text { hammer results }\end{array}$} & \multicolumn{3}{|c|}{ High-speed camera results } & \multicolumn{2}{|c|}{ FEA results } \\
\hline & & & & $\begin{array}{l}\text { Measured } \\
\text { voltage }^{\mathrm{a}} \\
\text { (V) }\end{array}$ & $\begin{array}{l}\text { Impact } \\
\text { force }^{b} \\
(\mathrm{~N})\end{array}$ & $\begin{array}{l}\text { Impact } \\
\text { velocity }^{\mathrm{b}} \\
\left(\mathrm{ms}^{-1}\right)\end{array}$ & $\begin{array}{l}\text { Kinetic } \\
\text { energy }^{b} \\
(\mathrm{~J})\end{array}$ & $\begin{array}{l}\text { Impact } \\
\text { force }^{b} \\
\text { (N) }\end{array}$ & $\begin{array}{l}\text { Impact } \\
\text { velocity }^{\mathrm{b}} \\
\left(\mathrm{ms}^{-1}\right)\end{array}$ & $\begin{array}{l}\text { Impact } \\
\text { force }^{\mathrm{b}} \\
\text { (N) }\end{array}$ \\
\hline 1 & 0.212 & 0.8065 & 0.0065 & $\begin{array}{l}2.08 \text { (refer } \\
\text { Fig. } 8 \text { ) }\end{array}$ & 4168 & 7.17 & 25.70 & 3954.5 & 7 & 3783 \\
\hline 2 & 0.210 & 0.8037 & 0.0037 & $\begin{array}{l}2.64 \text { (refer } \\
\text { Fig. } 8 \text { ) }\end{array}$ & 5290.5 & 6.02 & 18.1 & 4897.35 & 5.91 & 4721 \\
\hline
\end{tabular}

${ }^{\mathrm{a}}$ Measured values

${ }^{\mathrm{b}}$ Calculated values

Applying the same hammering data (hammer travelling for a distance of $0.8065 \mathrm{~m}(D+d)$ to produce a deformation of $0.0065 \mathrm{~m}(d)$ in $0.212 \mathrm{~s})$ in FEA simulation, performing numerical analysis, an impact velocity of $7 \mathrm{~ms}^{-1}$ was calculated for deforming the sheet metal to $0.0065 \mathrm{~m}$. The impact force was calculated as $3783 \mathrm{~N}$.

\subsection{Experiment 2}

The set-up was similar to the one discussed above, and the impact hammer of mass $1 \mathrm{~kg}$ was used to impact the sheet metal. The hammer travelled $0.210 \mathrm{~s}(t)$ for a distance of $0.8037 \mathrm{~m}(D+d)$ producing a deformation, $d$, of $0.0037 \mathrm{~m}$. The impact velocity of the hammer was determined by the high-speed camera and was calculated using Eq. 3 as $6.02 \mathrm{~ms}^{-1}$. The impact kinetic energy was calculated using Eq. 5 as $18.1 \mathrm{~J}$. The impact force was calculated using Eq. 6 as $4897.35 \mathrm{~N}$.

Similarly, using the impact hammer, a voltage of $2.64 \mathrm{~V}$ (refer Fig. 8-Exp2) was measured upon impact. Calculating the impact force based on the sensitivity of $0.499 \mathrm{mV} \mathrm{N}^{-1}$, the impact force was calculated as $5290.5 \mathrm{~N}$.

Applying the same hammering data (hammer travelling for a distance of $0.8037 \mathrm{~m}(D+d)$ to produce a deformation of

\section{Experiments 1 \& 2}

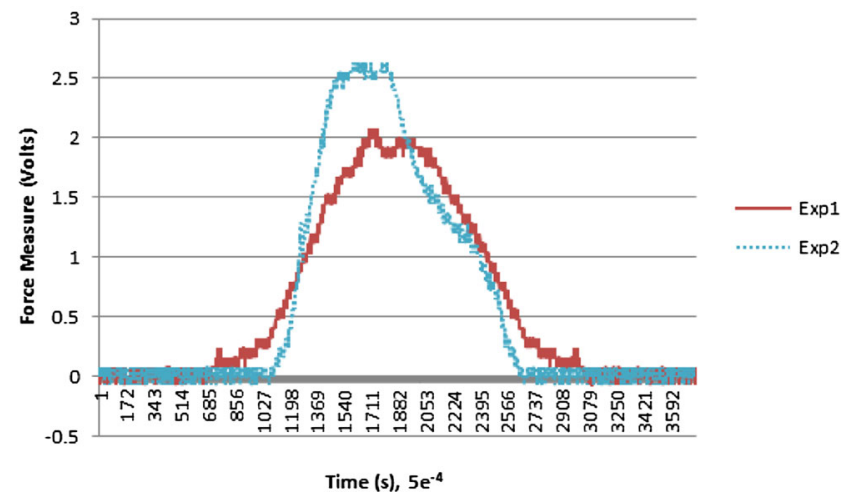

Fig. 8 Impact hammer data
$0.0037 \mathrm{~m}(d)$ in $0.210 \mathrm{~s}$ in FEA simulation, performing numerical analysis, an impact velocity of $5.91 \mathrm{~ms}^{-1}$ was calculated for deforming the sheet metal to $0.0037 \mathrm{~m}$. The impact force was calculated as $4721 \mathrm{~N}$.

\subsection{Discussion of experimental results}

Comparing experiments 1 and 2 (refer to Table 1), the impact force applied in experiment 1 is less than the impact force produced in experiment 2 but the deformation produced is larger in experiment 1 when compared to experiment 2 . This is because deformation depends on the impact kinetic energy. Higher impact kinetic energy produces larger deformation (experiment 1), similarly, smaller impact kinetic energy produces smaller deformation (experiment 2). This can also be observed from the area under curve of the voltage measured for experiments 1 and 2. By inspection, the area under the curve for experiment 1 is larger than the area under the curve for experiment 2 , suggesting that higher impact kinetic energy produces higher deformation and vice versa. However, the impact force applied depends on angular contact with the surface of the support based on the support's size and shape (refer Fig. 6).

Comparing the results, it could be observed that the impact force found using three methodologies (i.e. impact hammer, high-speed video and FEA) are similar with an acceptable margin of error.

Therefore, both experiments provide proof of concept on predicting the magnitude of the impact force required for incremental deformation of the part used in these experiments. Such prediction is then used to specify a pattern of part movement and related impact forces required to achieve the final deformation of parts.

\section{Human skill capture for database}

After determining the force required for sheet metal forming using the experiments discussed in Sect. 5, an impacting 
hammer capable of producing the force required was used as part of an integrated automated forming system. The hammering machine, Eckold Kraftformer [22], is pneumatically operated and was initially controlled using a foot pedal operation. After careful analysis, a dome shaped hammering tool (positive) with its anvil (negative) was used to form the sheet metal. The sheet metal forming process requires a human operator to manipulate the sheet metal blank between the hammer and the anvil during automatically repetitive hammering to progressively form the desired $3 \mathrm{D}$ shape. This skill of a human operator was captured and interpreted using a Vicon System [23].

\subsection{Human skill capture using Vicon System}

To understand and document the human behaviour during the hammering process, it was necessary to capture the motion of workpiece at six axes of freedom, in addition to the force and the frequency of the hammering. Two Vicon T-series [24] 2 megapixel cameras were used to interpret the human motion. The Vicon cameras were connected to the PC host through giganet Ethernet switch for fast data transfer.

As shown in Fig. 9, trackers were affixed on the hammer and sheet metal to recognise the objects in the Vicon environment using the tracker analyser. The motion of the hammer and sheet metal manipulation data were streamed out into Matlab environment using Simulink [25]. The data was processed in Matlab to obtain significant information necessary to automate the process. Figure 9 shows the manipulation path (3D) information acquired using Vicon system and Matlab processing. The coloured dots in Fig. 9 represent the hammering impact points relative to the manipulation path.

\subsection{Experimental tests and results of human skill capture}

The experiments were aimed at determining the system parameters discussed in Sect. 3.1. As used in the previous experiments, an aluminium sheet of $275-\mathrm{mm}$ diameter and $1-\mathrm{mm}$ thickness was used to form a bowl shape of 130-mm diameter. To reduce the variability factor and increase the accuracy in interpreting the sheet metal manipulation, the hammering sequence was fixed to produce a consistent impact force.

The cameras were calibrated for the experimental environment using the Vicon active wand [26]; hence, the universal position of hammer and the position of sheet metal relative to the hammer were identifiable. As the human operator used spiral, zig-zag, diagonal pattern to form the bowl shape, the live data was fed into the Vicon tracker. The captured data was processed in Matlab to analyse the system parameters. Based on the analysis performed in Matlab, the following results were obtained.

1. The sheet metal should always be held upon the anvil to ensure maximum impact force is produced by the hammering tool hence producing maximum deformation.

2. The spacing between the impacts were measured (through calculation) to be in the range of $5-10 \mathrm{~mm}$.

3. The $3 \mathrm{D}$ co-ordinates of sheet metal manipulation relative to hammer were obtained using Matlab. The results were in synchronisation with the time of hammering impact.

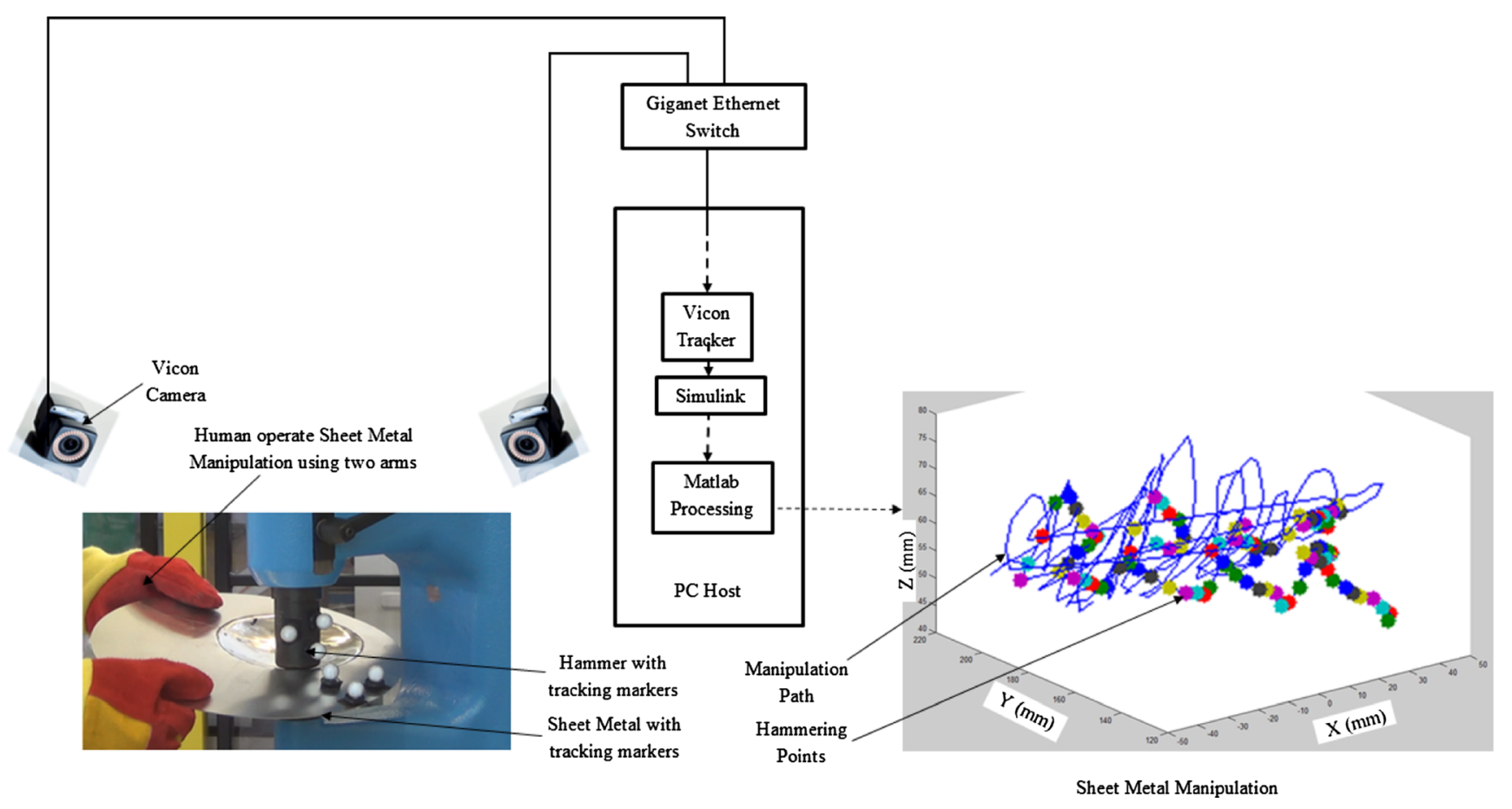

Fig. 9 Human manipulation interpretation using Vicon System and Matlab 
4. Results determined that the hammering machine was operating at a frequency of 4 impacts per second.

5. It was inferred from the results that the velocity of human manipulation was $5 \mathrm{~mm} \mathrm{~s}^{-1}$ during planar motion and $2 \mathrm{~mm} \mathrm{~s}^{-1}$ while skewing.

These results were significant in determining the system parameters necessary for developing a database of rules for the sheet metal manipulation in automated process - the database was influential in transferring the human skills of sheet metal manipulation to robotic panel movement. Experiments were also performed by affixing the markers in human arm to determine the factors of sheet metal gripping compliance. Based on the analysis, a gripper was designed to assist the robotic panel movement. Experiments are in progress to recognise the best pattern and the hammering path required to produce the bowl shape and the system parameters necessary in forming different contours.

\section{Robotic panel movement}

A robotic arm is being deployed to manipulate the work piece under the hammer in specific position and orientation. The motion will be calculated in (near) real time based on the feedback of existing contour captured by the vision system and the tool force required, calculated by the control algorithm in order to achieve a particular shape. It was discussed that the sheet metal will have to be moved along the $X Y$ plane and skewed about $X$ or $Z$ axis. Considering the complexity in prediction of forming (i.e. real-time processing power), the motion of the sheet metal is initially limited to simple physical shapes. Therefore, the robotic manipulation of sheet metal parts will have the motion on $X Y$ plane and rolling (rotation about $Z$ axis) and pitching (rotation about $X$ axis).

As part of on-going research in this project, the integration of the robotic arm with the proposed Mechatroforming concept (see Fig. 2) is completed and initial test in control environments has been successfully completed.

To continue this research further, tests are being developed to validate (a) the development of the physical geometry, (b) the dimensional accuracy and surface quality of the final parts, and (c) the performance of the system in real time. The research work is being continued to also formalise a method of automated impacting pattern to minimise the residual stresses, released in the form of distortion and spring back.

\section{Conclusions}

This research was aimed at understanding manual forming of sheet metal parts by skilled panel beaters and investigating the possibility of automating such operation.
By analysing the manual operations, a set of impacting pattern was captured in relation with the angle of the impact hammer and the manual motion of the parts. Mechatroforming method was proposed as a new automated forming approach for sheet metal parts. In this method, the incremental deformations of parts are monitored frequently by vision systems as a part of the proposed method to facilitate a (near) real-time control of the impacting processes. The incremental deformations of parts are predicted through FEA modelling to design a sequence of impacts to achieve the desired geometry.

The magnitude of the force required (i.e. the impact) for each incremental deformation was tested by two experiments designed to monitor the impact force of the hammer and measuring individual dents caused by the impact. The experiments were performed using instrumented hammer, time-based analysis, and FEA modelling to find the impact force for the same pre-defined deformation. By comparing the impact force achieved using each experiment, the results validated a consistency in the impact forces in both the experiments and the developed FEA model.

The results were found to comply with the force feedback provided by the force transducer. On this basis, it was proved that the prediction model (FEA model) can be used as a closed loop monitoring the impacts during forming the parts.

Unlike to the traditional method, in Mechatroforming method, the hammering direction was kept in vertical orientation and the part was manipulated by a robotic arm to locate the exact hammering position with correct orientation.

As the deformation was also predicted on a time based approach, it has led to the conclusion that using the proposed mechatronics to the controlled forming can potentially optimise the processes through an in-process monitoring and control system.

Acknowledgments The authors acknowledge support from the EPSRC Centre for Innovative Manufacturing in Intelligent Automation in undertaking this research work under grant reference number EP/IO33467/1.

Open Access This article is distributed under the terms of the Creative Commons Attribution 4.0 International License (http:// creativecommons.org/licenses/by/4.0/), which permits unrestricted use, distribution, and reproduction in any medium, provided you give appropriate credit to the original author(s) and the source, provide a link to the Creative Commons license, and indicate if changes were made.

\section{References}

1. Echrif SBM, Hrairi M (2011) Research and progress in incremental sheet forming processes. Mater Manuf Process 26(Issue 11):1404 1414, ISSN 1042-6914

2. Young D, Jeswiet J (2004) Wall thickness variations in single-point incremental forming. Proc Inst Mech Eng B J Eng Manuf 218(Issue 11):1453-1459, ISSN 0954-4054

3. Ben Hmida R, Thibaud S, Gilbin A, Richard F (2013) Influence of the initial grain size in single point incremental forming process for 
thin sheets metal and microparts: experimental investigations. Mater Des 45:155-165. doi:10.1016/j.matdes.2012.08.077, ISSN 0261-3069

4. Yang ZR, Scherer D, Golle M, Hoffmann H (2011) Geometrical modeling of the sheet metal parts in the incremental shrinking process. Key Eng Mater 473:509-515. doi:10.4028/www.scientific. net/KEM.473.509

5. Behera AK, Lauwers B, Duflou JR (2012) Advanced feature detection algorithms for incrementally formed sheet metal parts. Trans Nonferrous Metals Soc China 22(Issue 12):s315-s322. doi:10. 1016/S1003-6326(12)61725-7, ISSN 1003-6326

6. Silva M, Martins P (2013) Two-point incremental forming with partial die: theory and experimentation. J Mater Eng Perform 22(Issue 4):1018-1027

7. RADU C (2012) Analysis of the correlation accuracy-distribution of residual stresses in the case of parts processed by SPIF. 14th Mathematical models and methods in modern science, July 1-3, Portugal

8. Chung W, Cho J, Belytschko T (1998) On the dynamic effects of explicit FEM in sheet metal forming analysis. Eng Comput 15(Issue 6):750-776, ISSN 0264-4401

9. Meier H, Buff B, Smukala V (2009) Robot-based incremental sheet metal forming - increasing the part accuracy in an automated, industrial forming cell. Key Eng Mater 410-411:159-166. doi:10. 4028/www.scientific.net/KEM.410-411.159

10. Dieless NC Forming. AMINO North America Corporation, United States. [viewed 20.02.2014]. Available from: http://www. aminonac.ca/technology_dnc.asp

11. Freeform Fabrication Technology. FORD, United States. [viewed 20.02.2014]. Available from: https://media.ford.com/content/ fordmedia/fna/us/en/news/2013/07/03/ford-develops-advancedtechnology-to-revolutionize-prototyping-.html

12. Jeswiet J, Micari F, Hirt G, Bramley A, Duflou J, Allwood J (2005) Asymmetric single point incremental forming of sheet metal. CIRP Ann Manuf Technol 54(Issue 2):88-114, ISSN 0007-8506

13. Kreimeier D, Buff B, Magnus C, Smukala V, Zhu J (2011) Robotbased incremental sheet metal forming - increasing the geometrical accuracy of complex parts. Key Eng Mater 473:853-860
14. Tanaka H, Naka S, Asakawa N (2012) Development of CAM system using linear servo motor to automate metal hammering - a study on forging-type rapid prototyping system. J Ref Int J Autom Technol 6(Issue 5):604-610

15. Schafer T, Schraft RD (2005) Incremental sheet metal forming by industrial robots. Rapid Prototyp J 11(Issue 5):278-286

16. Opritescu D, Sachnik P, Yang Z, Golle R, Volk W, Hoffmann H, Schmiedl F, Ritter M, Gritzmann P (2012) Automated driving by standardizing and scaling the manufacturing strategy. Procedia CIRP 3:138-143

17. CoDrive. Costesys, Germany. http://cotesys-v2.in.tum.de/research/ list-of-projects.html?projectid $=33 \& \mathrm{cHash}=\mathrm{e} 8 \mathrm{aa} 83 \mathrm{dcdf}$. Accessed 21 Feb 2014

18. Scherer D, Yang Z, Hoffmann H (2010) Driving-a flexible manufacturing method for individualized sheet metal products. Int J Mater Form 3:955-958. doi:10.1007/s12289-010-0927-5, ISSN 1960-6206

19. Innovative Sheet Forming Processes. University of Cambridge, UK. http://www.lcmp.eng.cam.ac.uk/wellformed/innovativesheet-forming-processes. Accessed 21 Feb 2014

20. Nave CR (2014) HyperPhysics (Mechanics). http://hyperphysics. phy-astr.gsu.edu/hbase/avari.html. Accessed 17 Apr 2014

21. Goldsmith W (2001) Impact: the theory and physical behaviour of colliding solids. Dover, Mineola. ISBN 9780486420042

22. Eckold (2015) Kraftformer KF 170 PD. http://www.eckold.com/ enus/productsforsheetmetalworking/kraftformer/ kraftformerkf170pd.aspx. Accessed 5 Feb 2015

23. Vicon (2015). Vicon System. http://www.vicon.com/. Accessed 14 Apr 2015

24. Vicon (2015) Vicon T-Series. http://www.vicon.com/System/ TSeries. Accessed 10 Apr 2015

25. Matlab (2015) Simulink. http://uk.mathworks.com/products/ simulink/. Accessed 14 Apr 2015

26. Vicon (2015) Vicon active wand. http://www.vicon.com/System/ Calibration. Accessed 10 Apr 2015 